\title{
Set Distributed Path by the Network Load in Mobile Ad-hoc Networks
}

\author{
Dong-keun Oh, Young-jun Oh and Kang-whan Lee \\ Department of Computer Science Engineering, Interdisciplinary Program in \\ Creative Engineering Korea University of Technology and Education \\ Department of Computer Science Engineering, Korea University of Technology \\ and Education \\ Department of Computer Science Engineering, Interdisciplinary Program in \\ Creative Engineering Korea University of Technology and Education \\ shyawing@koreatech.ac.kr
}

\begin{abstract}
Mobile Ad-hoc Network (MANET) consists of a node that has mobility. In MANET, node function as routing. So they build a network of their own, no infrastructure. Topology is exchanged due to node mobility in MANET. For reducing changed topology, hierarchical network algorithms are researched. However load-balancing of cluster head node is exceeded, assigned cluster member node can't communicate other node. To solve this problem, we proposed Load Tolerance (L.T) algorithm for distributing routing. Proposed algorithm, when the cluster head node is measured overflow load balancing, cluster member node sends a message through distributed routing path by LoadTolerance node. As a result, the proposed algorithm improves the packet delivery ratio in cluster routing
\end{abstract}

Keywords: Distributed routing path, Load balancing, Mobile Ad-hoc network, Cluster

\section{Introduction}

Mobile Ad-hoc Network (MANET) consists of a node that has mobility. In MANET, each node function as routing. So they build a network of their own, no infrastructure. Topology is frequently exchanged due to node mobility in MANET, so load balancing is exchanged. For reducing frequently exchanged topology, hierarchical networks are researched. Hierarchical is the network that forms group for reducing exchange topology[1]. Exchange topology is reduced by the hierarchical network in MAENT, the network uses efficient energy consumption and has a stable load balancing[2][3]. However load balancing of certain cluster head node is concentrated, assigned cluster member node can't communicate other node[4]. To solve this problem, we proposed Load Tolerance algorithm. Proposed algorithm, when cluster head node, upper level node, is concentrated transmit of packet, storage of packet is overflow, cluster member node, lower child node, measures load balancing of cluster head nodes in efficient communication coverage. When an assigned cluster member node checks that it's cluster head node is concentrated traffic, cluster member node makes distributed routing path using a property of nodes like distance between nodes, energy, direction, velocity and communicates to other node through distributed routing path.

\section{Related Work}

The existing cluster routing algorithm keeps the connection between nodes in MANET, So they reduced overhead, kept communication. However, certain cluster head is concentrated packet, an assigned cluster member node can't send message to 
cluster head node and another node. In RODMRP(Resilient Ontology-based Dynamic Multicast Routing Protocol) algorithm, the network composed of tree formation, a lower level node has step parent node. When a lower level node can't communicate with its upper level node, it communicates using a step parent node. In this paper, assigned lower level node measures upper level node load balancing. When the lower level node checks that load balancing of its upper level node occurs overflow, proposed algorithm resets routing path using load-tolerance(L.T) node.

DDV (Dynamic Distance Vector) algorithm, the proposed algorithm in reference work, forms and maintains cluster formation using the direction and velocity of property, the algorithm reduces change routing path by change topology and keeps communication. In DDV algorithm, cluster formation is formed by a threshold value of direction and velocity that is made by the base-station. In this paper, for selecting L.T node, we use difference of direction between nodes that suggested in DDV algorithm. Moreover, we calculate probability using other property of node for selecting L.T node.

\section{System Model and Methods}

The proposed L.T algorithm is which assigned cluster member node checks traffic of its cluster head node. When a cluster head node is concentrated load balancing, its cluster member node set distributed routing path of the selected L.T node. In L.T algorithm, showing the routing path, we define the relationship between nodes. The relationship of the cluster member node and the cluster head node is as follows.

$$
\begin{array}{r}
\mathrm{X}_{\mathrm{M}}=\left\{\mathrm{x}_{\mathrm{M}(\mathrm{i}, \mathrm{j})} \mid \mathrm{x}_{\mathrm{M}(\mathrm{i}, \mathrm{j})}=0 \text { or } 1\right\} \\
x_{M(i, j)}=\left\{\begin{array}{l}
1, \text { if node } j \text { is among cluster head node } i \\
0, \quad \text { otherwise }
\end{array}\right.
\end{array}
$$

As an equation (1) and (2) is show relationship of cluster head node between cluster member nodes. Where $X_{M}$ means set of relationship that is between cluster member node and cluster head node. $\mathrm{x}_{\mathrm{M}(\mathrm{i}, \mathrm{j})}$ shows the status of relationships like 0 or 1.1 means that the cluster head node i and cluster member node j. 0 means otherwise. After defining between cluster member node and cluster head node, we define the relationship between cluster head nodes. The relationship of cluster head nodes is as follows.

$$
\begin{aligned}
& \mathrm{X}_{\mathrm{H}}=\left\{\mathrm{x}_{\mathrm{H}\left(\mathrm{i}, \mathrm{i},{ }^{\prime}\right)} \mid 0 \leq \mathrm{x}_{\mathrm{H}\left(\mathrm{i}, \mathrm{i}^{\prime}\right)} \leq 1\right\} \\
& x_{H(i, j)}=\left\{\begin{array}{lr}
0<x_{H(i, j)} \leq 1, & \text { if cluster head node } i^{\prime} \text { is connect cluster head node } i \\
0, & \text { otherwise }
\end{array}\right.
\end{aligned}
$$

As an equation (3) and (4) means relationships of each cluster head node. Where $\mathrm{X}_{\mathrm{H}}$ means a set of relationship that is between cluster head nodes. And it has element $\mathrm{X}_{\mathrm{H}\left(\mathrm{i}, \mathrm{i}^{\prime}\right)}$. $\mathrm{x}_{\mathrm{H}\left(\mathrm{i}, \mathrm{i}^{\prime}\right)}$ shows the status of relationships as between 0 and 1 . Between 0 and 1 means that the cluster head node i connects cluster head node i'. The value has lower, the connection between cluster head node has disconnected probability higher. 0 means that otherwise.

For calculating load tolerance probability of cluster member nodes and doing load balancing of cluster head node, we set a coverage of node as follows.

$$
\mathrm{R}_{\mathrm{C}}=\mathrm{k} \times \mathrm{R}_{\mathrm{C}}, k=2
$$

Where $\mathrm{k}$ is number of relay hop, $\mathrm{R}_{\mathrm{T}}$ is the transmission range of nodes, and $\mathrm{R}_{\mathrm{C}}$ is coverage of nodes. We know attribute and communication of node by a number of relays hops[5]. 


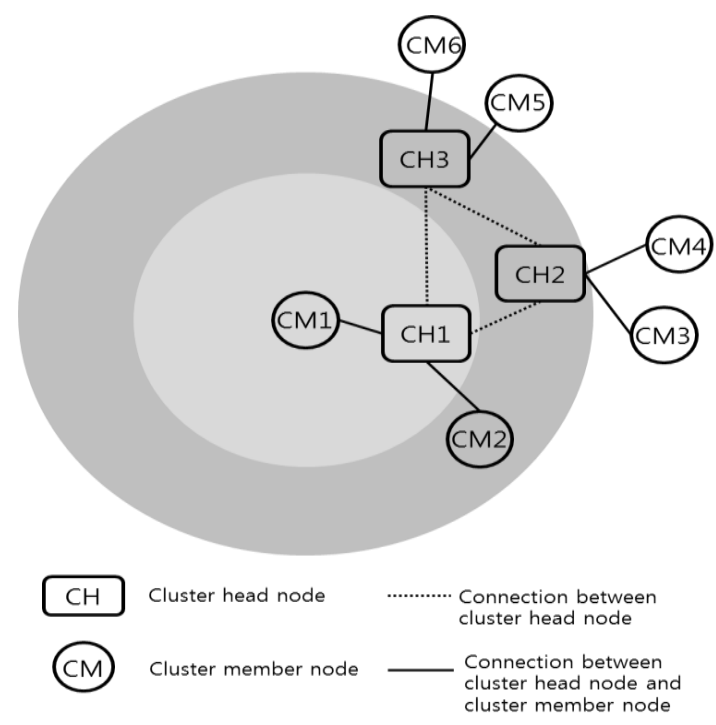

Figure 1. Example of Coverage of Node by the Hops

As shown in Figure 1, we assume the recovery coverage as number of $\mathrm{k}=2$ in reference work. So cluster member node takes cognizance of assigned cluster head node besides other cluster head node.

\subsection{Set Load Tolerance node}

When communication coverage of node defines, cluster member node selects LoadTolerance(L.T) node for continuous communication. So the cluster member calculates probability of selecting L.T node using properties of node such as direction, velocity, distance between nodes and residual energy.

A method calculates probability using the distance between nodes, it is calculated by comparing the distance between nodes with communication coverage. An equation is as follows.

$$
\left.\mathrm{P}_{\mathrm{i}^{\prime}}(\mathrm{t})\right|_{\text {dist }}=\frac{\text { dist }_{\mathrm{i}^{\prime} \mathrm{j}}}{\left|\mathrm{R}_{\mathrm{C}}\right|}
$$

Here $\left.\mathrm{P}_{\mathrm{i}^{\prime}}(\mathrm{t})\right|_{\text {dist }}$ means probability, by the distance between nodes, dist ${ }^{{ }^{\prime} \mathrm{j}}(\mathrm{t})$ means the distance between cluster head node i' and cluster member node $\mathrm{j}$, when time is $\mathrm{t}$.

A method calculates probability using direction, it is calculated using difference of direction in DDV algorithm, an equation is as follows.

$$
\left.\mathrm{P}_{\mathrm{i}^{\prime}}(\mathrm{t})\right|_{\text {Dir }}=\frac{\operatorname{Dir}_{\mathrm{i}^{\prime} \mathrm{j}}}{\left|\operatorname{Dir}_{\mathrm{j}}^{\mathrm{rs}}\right|}
$$

Here, $\left.\mathrm{P}_{\mathrm{i}^{\prime}}(\mathrm{t})\right|_{\text {Dir }}$ means probability, by direction, $\operatorname{Dir}_{\mathrm{i}^{\prime} \mathrm{j}}$ means the difference between cluster head node i' and cluster member node $\mathrm{j}$. Dir ${ }_{j}^{\text {rs }}$ means the threshold of difference of direction[6].

A method calculates probability using residual energy, it is calculated by comparing residual energy and initial energy, an equation is as follows.

$$
\left.\mathrm{P}_{\mathrm{i}^{\prime}}(\mathrm{t})\right|_{\mathrm{E}}=\frac{\mathrm{E}_{\mathrm{i}^{\prime}}(\mathrm{t})}{\left|\mathrm{E}_{\text {init }}\right|}
$$

Here, $\mathrm{P}_{\mathrm{i}^{\prime}},(\mathrm{t}) \mid \mathrm{E}$ means probability, by residual energy, $\mathrm{E}_{\mathrm{i}^{\prime}}(\mathrm{t})$ means residual energy when time is $t$. $E_{\text {init }}$ means initial energy.

For calculating probability of load balancing, load balancing define by capacity and flow of the node. 


$$
\mathrm{lb}_{\mathrm{i}}(\mathrm{t})=\frac{\text { flow }_{\mathrm{i}}(\mathrm{t})}{\mathrm{C}_{\mathrm{i}}} \times \frac{1}{\mathrm{C}_{\mathrm{i}}^{\alpha}}, \alpha=1,2,3, \ldots
$$

Here, $l b_{i}(t)$ means load balancing of node, when time is t. flow $(t)$ means the amount of flow packet in node i. $\mathrm{C}_{\mathrm{i}}$ means a capacity of node $\mathrm{i}$ for storing packet. $\mathrm{C}_{\mathrm{i}}{ }^{\mathrm{a}}$ means a condition of node by condition of network, the value is different by constant number $\mathrm{a}$. A load balancing has a value between 0 and 1 . the value is higher, the mount of storage of node $\mathrm{i}$ is smaller. A method calculates probability using load balancing, it is as follows.

$$
\left.\mathrm{P}_{\mathrm{i}^{\prime}}(\mathrm{t})\right|_{\mathrm{lb}}=1-P_{i^{\prime}}(t)
$$

Here, $\mathrm{P}_{\mathrm{i}},\left.(\mathrm{t})\right|_{\mathrm{b}}$ means probability, by load balancing.

For calculating select L.T node probability, multiply each probability of property by each weight of property. An equation is as follows.

$$
\begin{aligned}
& \left.\mathrm{P}_{\mathrm{i}^{\prime}}(\mathrm{t})\right|_{\mathrm{L} . \mathrm{T}}=\left|\begin{array}{c}
\left.\mathrm{P}_{\mathrm{i}^{\prime}}(\mathrm{t})\right|_{\text {dist }} \times \omega_{\mathrm{i}^{\prime}}(\text { dist })+\left.\mathrm{P}_{\mathrm{i}^{\prime}}(\mathrm{t})\right|_{\mathrm{E}} \times \omega_{\mathrm{i}^{\prime}}(\mathrm{E}) \\
+\left.\mathrm{P}_{\mathrm{i}^{\prime}}(\mathrm{t})\right|_{\text {Dir }} \times \omega_{\mathrm{i}^{\prime}}(\text { Dir })+\left.\mathrm{P}_{\mathrm{i}^{\prime}}(\mathrm{t})\right|_{\mathrm{lb}} \times \omega_{\mathrm{i}^{\prime}}(\mathrm{lb})
\end{array}\right| \\
& \omega_{\mathrm{i}^{\prime}}(\mathrm{attr})=\left\{\omega_{\mathrm{i}^{\prime}}(\text { dist })=\beta, \omega_{\mathrm{i}^{\prime}}(\mathrm{E})=\beta, \omega_{\mathrm{i}^{\prime}}(\text { Dir }), \omega_{\mathrm{i}^{\prime}}(\mathrm{lb})\right\}
\end{aligned}
$$

Here, i means cluster head node, j means cluster member node. i' means cluster head node that is located in communication coverage of cluster member node $j . P_{i}(t) \mid L . T$ means the probability of selection L.T node. $\omega_{\mathrm{i}^{\prime}}(\mathrm{dist}), \omega_{\mathrm{i}^{\prime}}(\mathrm{Dir}), \omega_{\mathrm{i}^{\prime}},(\mathrm{E}), \omega_{\mathrm{i}^{\prime}}(\mathrm{lb})$ mean weight of each property. An equation (11) means, node has various properties, we consider various node's condition. When node's property has a relationship of attention, the sum of node's property is 1 . For example, distance and energy have the relationship, sum of weight value of distance and the weight value of energy must 1 . When a cluster head node has a probability of select L.T node in a communication of cluster member node, the cluster member node chooses the cluster head node as L.T node that has maximum probability.

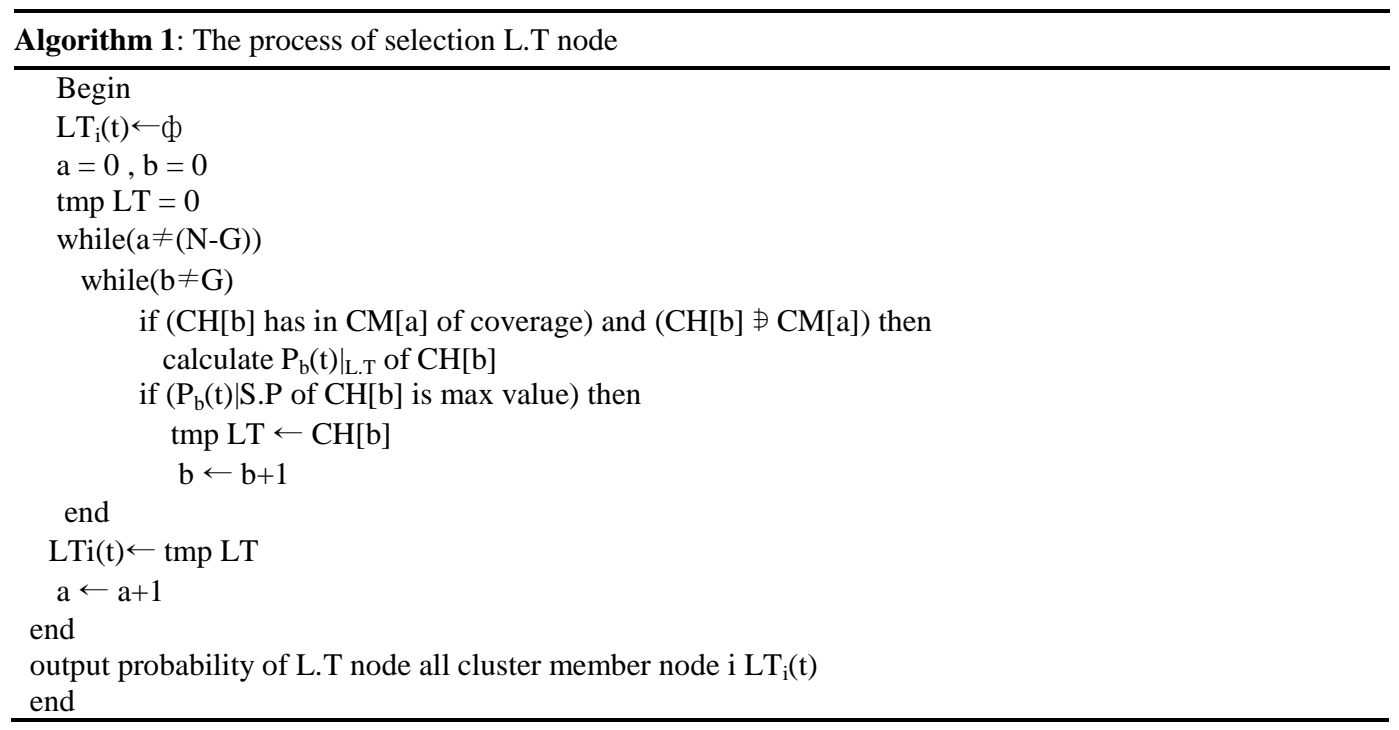

For selection L.T node, the cluster member node searches the cluster head node in communication coverage and calculates the probability of selection L.T node. When a cluster head node has maximum probability, the cluster head node is selected L.T node.

\subsection{Set Distributed Routing Path}

When a relationship of nodes defines by an equation (1) (4), routing path of each node expressed as an equation (13) and Table 1.

$$
\text { Path }_{(\mathrm{j}, \text { Des })}=\left\{\mathrm{x}_{\mathrm{M}(\mathrm{i}, \mathrm{j})}, \mathrm{x}_{\mathrm{H}(\mathrm{i}, \text { Des })} \mid \mathrm{x}_{\mathrm{M}(\mathrm{i}, \mathrm{j})} \cup \mathrm{x}_{\mathrm{H}(\mathrm{i}, \text { Des })}\right\}
$$


Table 1. The Table of Routing Path

\begin{tabular}{lcccccccc}
\hline Priority & S & $\mathbf{1}$ & $\mathbf{2}$ & $\mathbf{3}$ & $\ldots$ & $\ldots$ & Des-1 & Des \\
\hline Node ID & $\#$ & CH3 & CH4 & CH6 & $\ldots$ & $\ldots$ & CH9 & $\#$ \\
\hline
\end{tabular}

Here, Des means destination node, S means source node. In equation (13), routing path between a source node and destination node are defined by the relationship between nodes.

For continuous communication, cluster member node stores load balancing and probability of selection L.T of the cluster head node that is located in communication coverage in status table. The status table is expressed as an equation (14) and Table 2.

$$
\text { Table } \left._{\text {status }(\mathrm{j})}=\left\{\mathrm{x}_{\mathrm{M}(\mathrm{i}, \mathrm{j})}, \mathrm{x}_{\mathrm{H}\left(\mathrm{i}, \mathrm{i}^{\prime}\right)}\right)\left|\mathrm{lb}_{i}, \mathrm{lb}_{i^{\prime}}, P_{i}(t)\right|_{L . T},\left.P_{i^{\prime}}(t)\right|_{L . T}\right\}
$$

Table 2. The Status Table of Cluster Member Node

\begin{tabular}{lcccc}
\hline CHID & CH1 & CH2 & CH3 & CH5 \\
\hline Load balancing & 0.54 & 0.96 & 0.10 & 0.38 \\
\hline $\mathrm{P}_{\left.\mathrm{i}(\mathrm{t})\right|_{\text {L.T }}}$ & 0.32 & 0.25 & 0.96 & 0.40
\end{tabular}

In the status table of cluster member node, when the cluster head node, assigns the cluster member node, has a minimum value of load balancing, cluster member node make a conclusion that cluster head node is concentrated traffic and set distributed routing path. The process of setting distributed routing path is expressed as Figure 2.

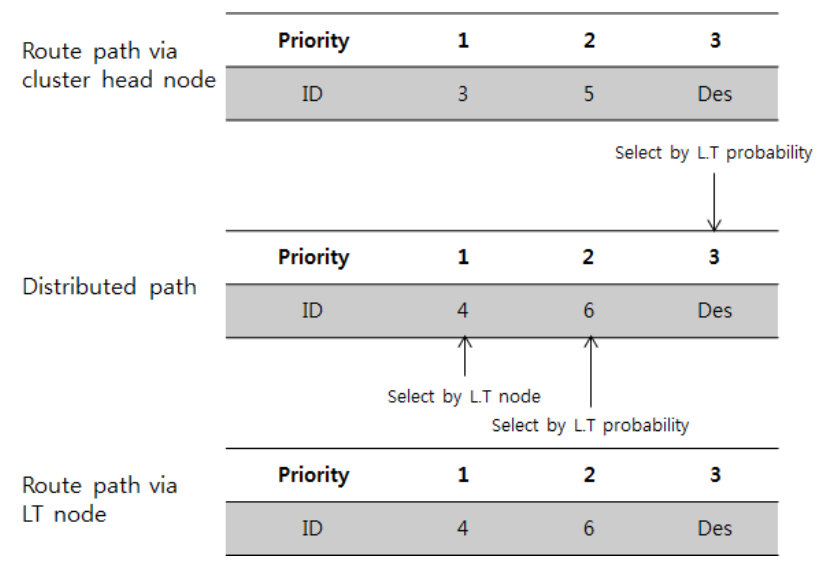

Figure 2. The Procedure of Setting Distributed Routing Path in the Routing Table

As shown in Figure 1, cluster member node forms routing path L.T node instead of its cluster head node. Since then, L.T node measure a probability of selection L.T node of other cluster head node in communication coverage, L.T node has chosen cluster head node that has the maximum probability of selection L.T node. The above process is repeated, when the routing path reaches destination node, cluster member node communicates using distributed routing path. The process of setting distributed routing path is expressed as an equation (16)

$$
\begin{aligned}
& \text { s.t } \mathrm{x}_{M(i, j)} \times l b_{i}(t) \text { is max value } \\
& \operatorname{D.Path}_{\left(j . j^{\prime}\right)}=\left\{X_{M}, X_{H}\left|x_{H\left(j, i^{\prime}\right)} \times \max P_{i^{\prime}}(t)\right|_{L . T}\right\}
\end{aligned}
$$

Here, $\boldsymbol{D . P a t h} \boldsymbol{~}_{\left(j \boldsymbol{j}^{\prime}\right)}$ means to distribute routing path source node $\boldsymbol{j}$ to destination node $\boldsymbol{j}$ '. The process of set distributed in the network is expressed as Figure 3. 


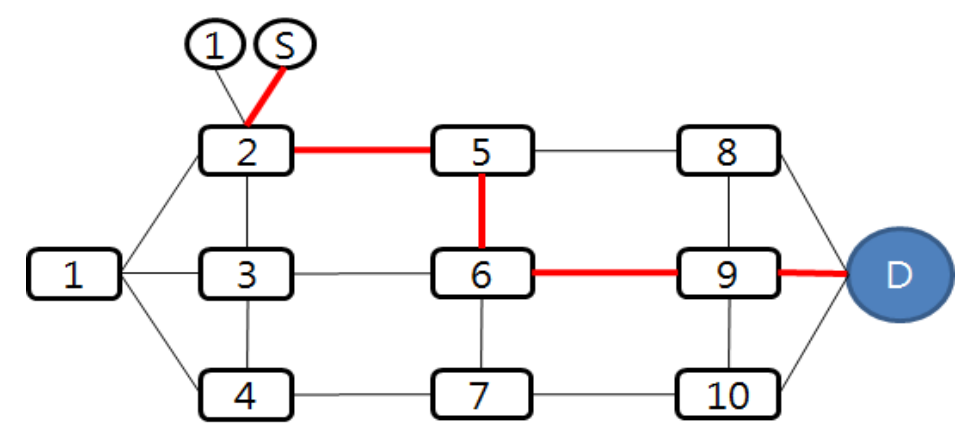

(a) Before Distributing Routing Path Network

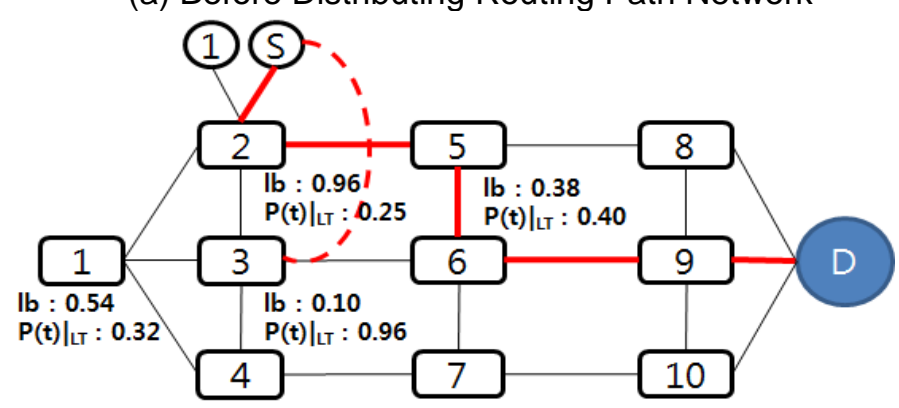

(b) Set Distributed Routing Path Using Status Table

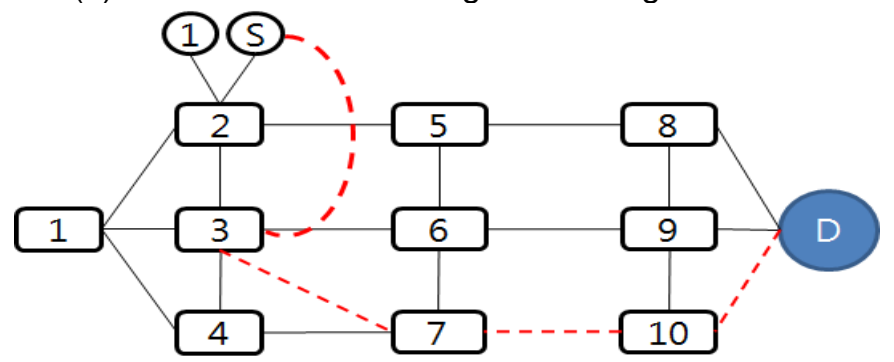

(c) Communicate Distributed Routing Path

Figure 3. Example the Process set Distributed Routing Path

As shown in Figure 2, $\mathrm{S}$ means source node that sends packet, $\mathrm{D}$ means destination node that receives the packet. Figure 2 (a) is the network that is not adopted distributed routing path, cluster member node sends packet to cluster head node. Figure 2 (b) is a process set distributed routing path, cluster member node measures load balancing of its cluster head node. When the cluster head node has a low value of load balancing, the cluster member node selects L.T node and sets distributed routing path. Since then, as shown in Figure 2 (c), cluster member node communicates using distributed routing path.

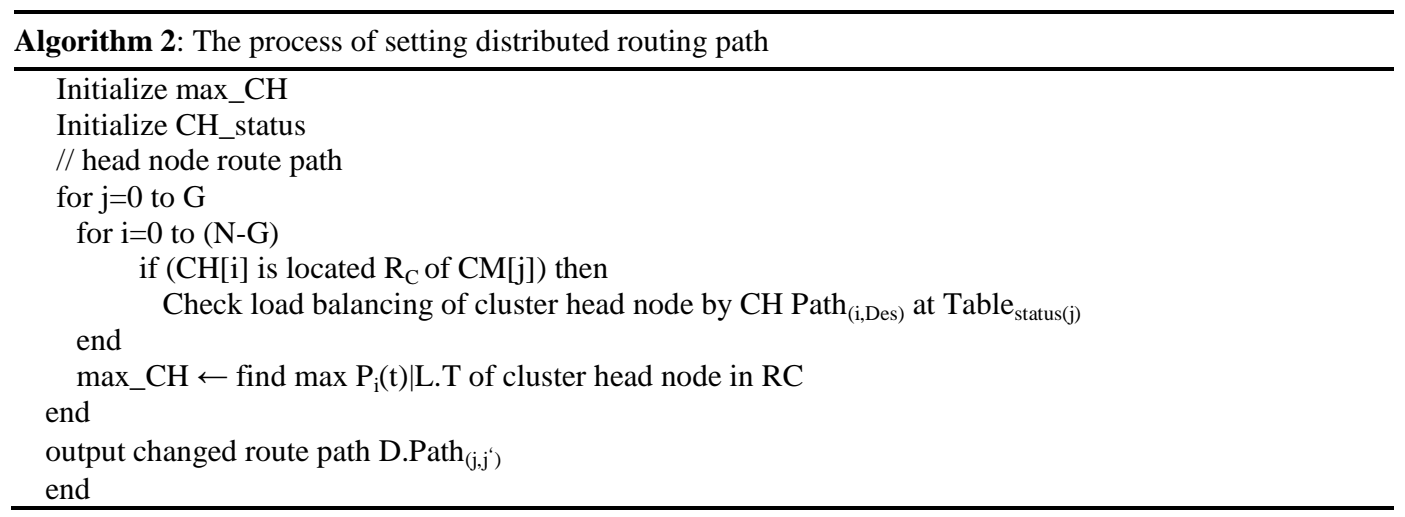


Here, for setting distributed routing path by L.T node, a cluster member node compares the probability of selection L.T node, a cluster head node, has the maximum probability of selection L.T node, is formed in the distributed routing path. Also selected cluster head node compares the probability of selection L.T node of other cluster head node in communication coverage, and chooses the cluster head node for setting distributed routing path.

\section{Simulation and Result}

Simulation evaluated LT algorithm that compares random cluster algorithm. Simulation parameter is as follows. The simulation time is 300 second. Simulation measures packet delivery ratio every 30 seconds.

Table 5. Simulation Configuration

\begin{tabular}{ll}
\hline Parameter & Value \\
\hline Area & $100 \times 100$ \\
\hline Velocity $(\mathrm{m} / \mathrm{s})$ & $1 \sim 16$ \\
\hline $\begin{array}{l}\text { Number of cluster head } \\
\text { nodes }\end{array}$ & 16 \\
\hline Number of nodes & $100,200,300,400$ \\
\hline Jammer range(m) & 30 \\
\hline Transmission cycle $(\mathrm{Sec})$ & 1 \\
\hline Pause time (Sec) & $30,60,90,120,150,180$, \\
\hline Simulation time (Sec) & 310,240 and 270 \\
\hline
\end{tabular}

Figure. 4 shows the packet delivery ratio about LT algorithm and random cluster algorithm. As shown in Figure. 5, Regardless of the number of nodes, LT algorithm holds the packet delivery ratio of $80 \sim 100 \%$. However, the random cluster algorithm holds the packet delivery ratio of $40 \sim 60 \%$. So we check what LT algorithm is higher than random cluster algorithm and is kept at a packet delivery ratio.

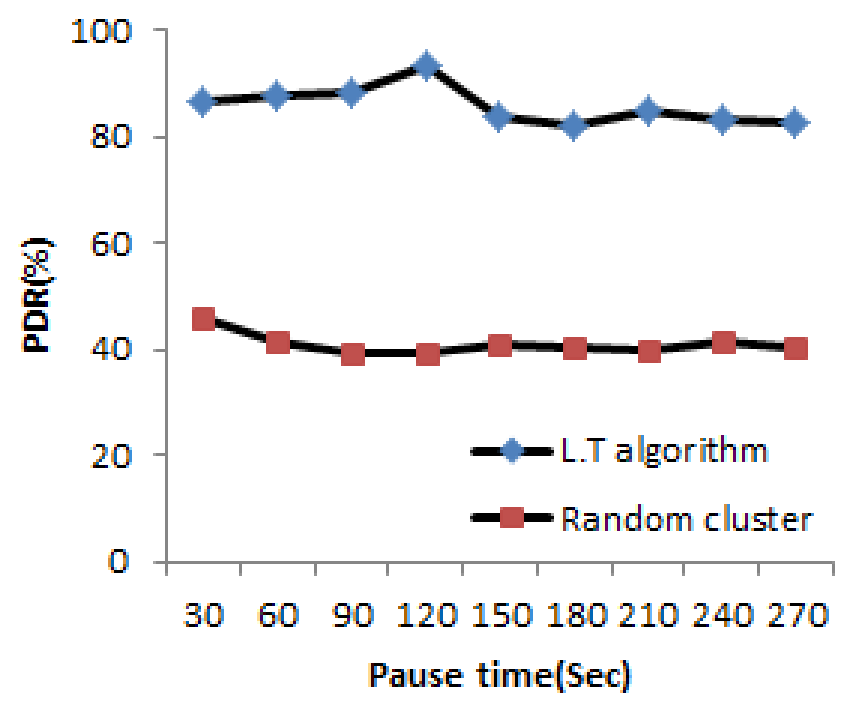

(a) number of nodes $=100$ 


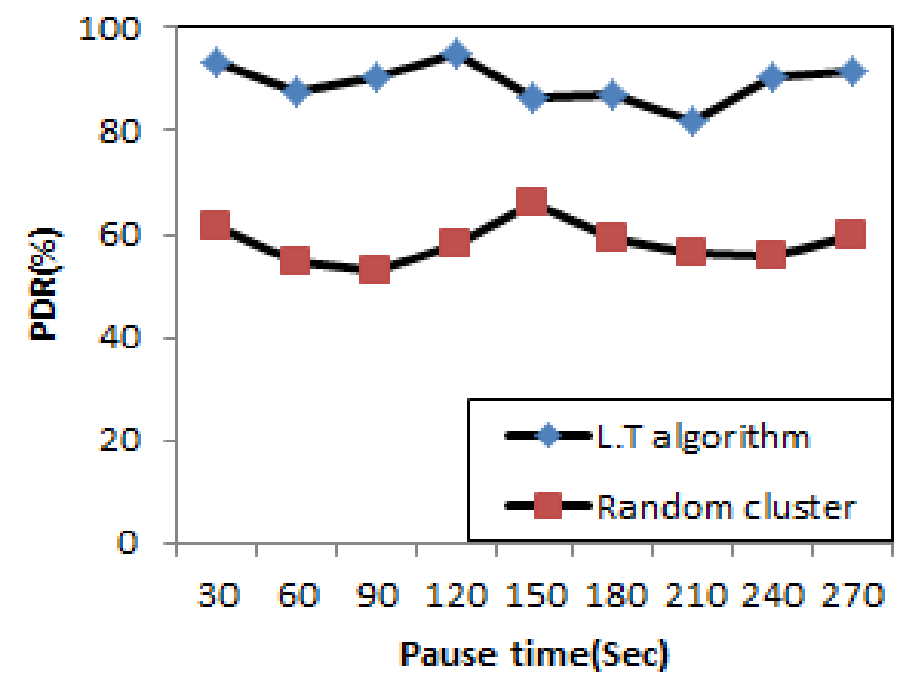

(b) number of nodes $=200$

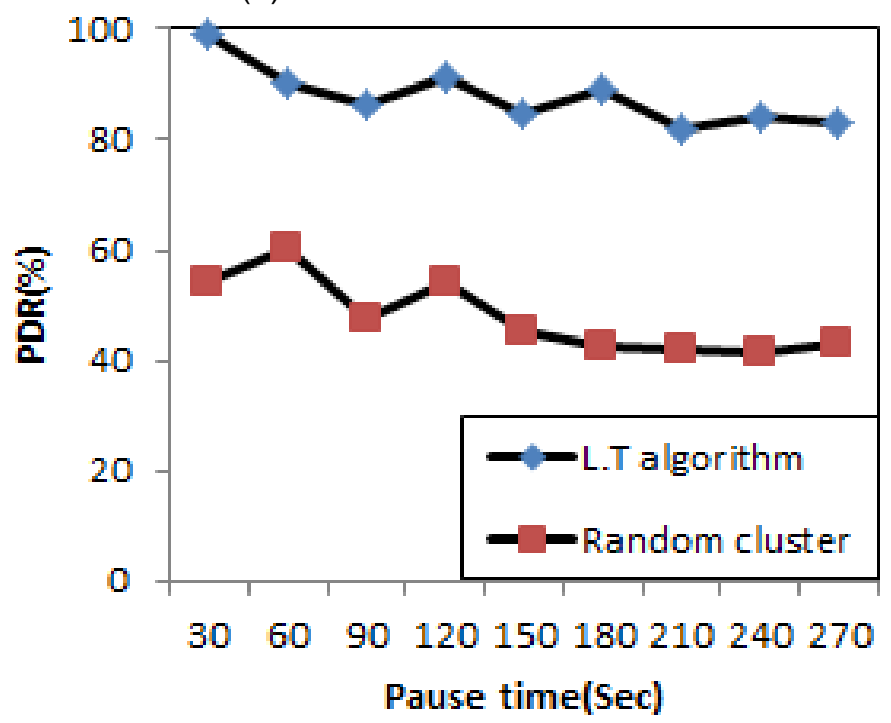

(c) number of nodes $=300$

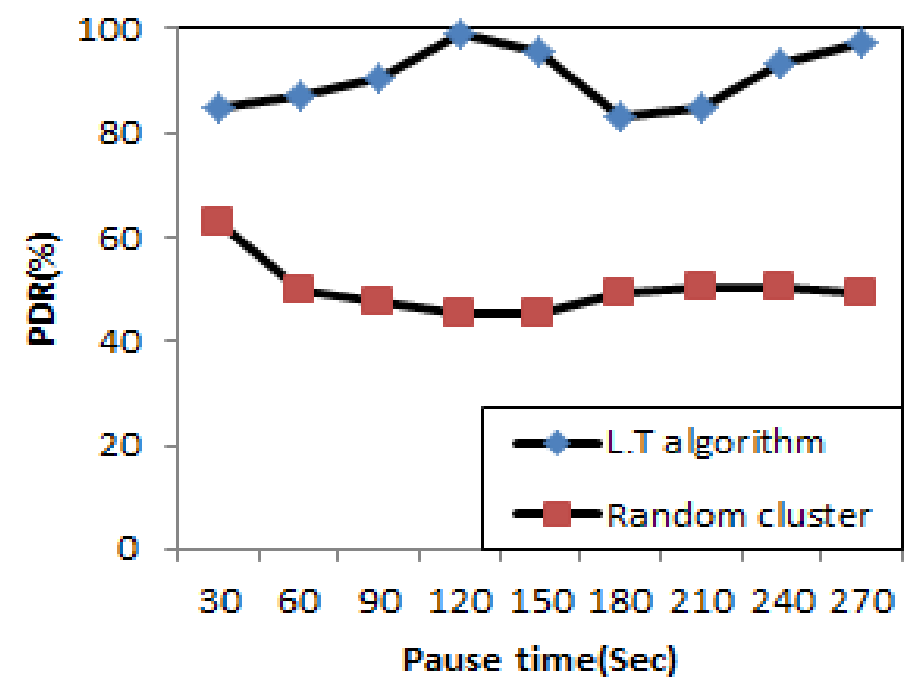

(d) number of nodes $=400$

Figure 5. Packet Delivery Ratio with LT Algorithm 


\section{Conclusions}

In this paper, we proposed LT algorithm. LT algorithm selects LT node by attribute of node such as direction, load balancing, and energy. When a cluster head node is concentrated packet, cluster member node maintain s communication using distributed routing by LT node. Simulation result, packet delivery ratio is higher than random cluster when the network has jammer or packet is concentrated in cluster head. According to network characteristics such as increasing the network area, many jammer, Research result is different. So we need more study considering the characteristics of the network.

\section{References}

[1] R. Agarwal and M. Motwani, "Survey of cluster of clustering algorithms", International journal on Computer Science and Engineering, vol. 1, no. 2, (2009).

[2] L. Lazos and M. Krunz, "Selective jamming/dropping insider attacks in wireless mesh network", IEEE Journals \& Megazines, vol. 25, (2011).

[3] S. G. Kim and K. W. Lee, "A study on the advanced inference routing network scheme for RODMR", International Conference on Advanced language Processing and Web Information Technology, (2008).

[4] Y. J. Oh and K. W. Lee, "Energy conserving routing algorithm based on the direction for Mobile Adhoc network", Proceedings Conference on Information and Communication Engineering, vol. 17, no. 1, (2013).

[5] G. Mitsuo, R. W. Cheng and L. Lin, "Backbone Network Models", Network Models and Optimization: Multiobjective Genetic Alogrithm Approach, Springer, (2008).

[6] J. J. Lee and J. S. Lim, "Effective and Efficient Jamming Based on Routing in Wireless Ad Hoc Networks", IEEE Communication Letters, vol. 16, no. 11, (2012).

[7] Y. J. Oh, D. K. Oh and K. W. Lee, "A Study Optimal Path Availability Clustering Algorithm in Ad Hoc Network", Future Information Communication Technology and Applications Lecture Notes in Electrical Engineering, vol. 235, (2013).

[8] J. J. Liu, X. H. Jiang, H. Nishiyama, R. Miura, N. Kato and N. kadowaki, "Optimal Forwarding Games in mobile Ad Hoc Networks with Two-Hop f-cast Relay", Selected Areas in Communications, IEEE Journal on, vol. 30, no. 11, (2012).

[9] P. Manickam, T. G. Baskar, M. Girija and D. Manimegalai, "Performance Comparisons of Routing Protocols in Mobile Ad Hoc Networks", International Journal of Wireless \& Mobile Networks (IJWMN), vol. 3, no. 1, (2011).

[10] W. Y. Xu, K. Ma, W. Trappe and Y. Y. Zhang, "Jamming Sensor Networks: Attack and Defense Strategies", Network, IEEE, vol. 20, no. 3, (2006).

[11] X. B. Wang, X. J. Lin, Q. S. Wang and W. T. Luan, "Mobility Increase the Connectivity of Wireless Networks", IEEE/ACM Transactions on Networking, vol. 21, no. 2, (2013).

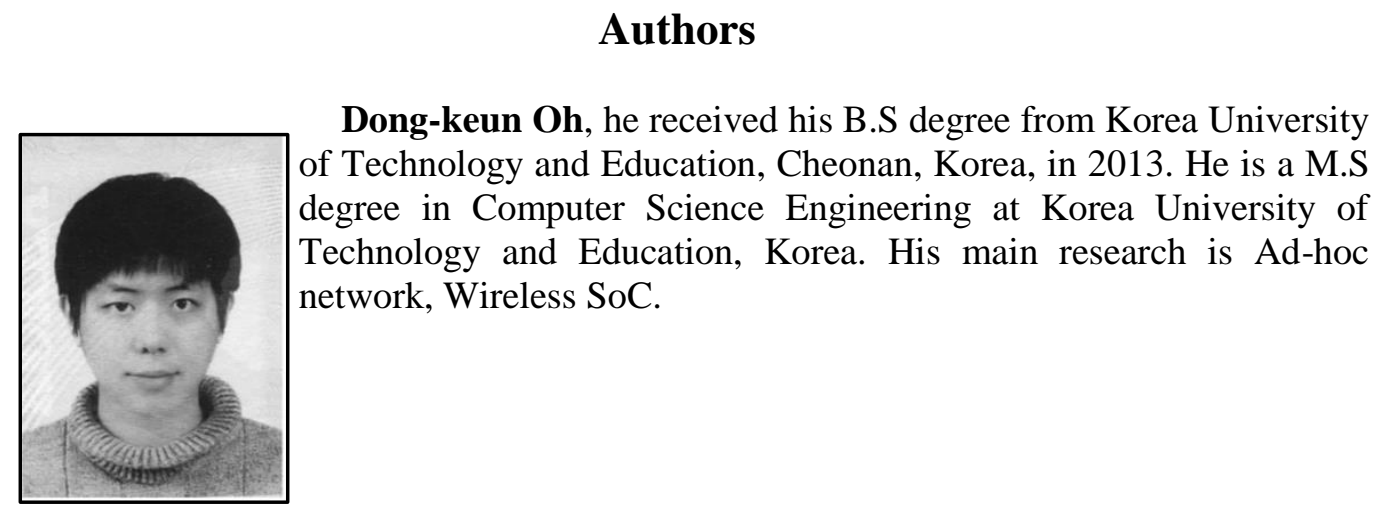




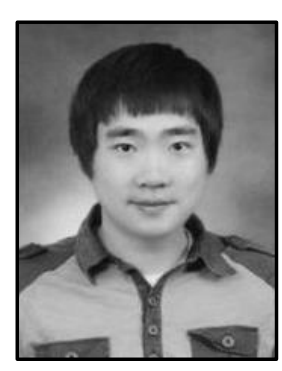

Young-jun Oh, he received his B.S degree from Nasalet University, Cheonan, Korea, in 2009. The M.S degree in electronics engineering from Korea University of Technology and Education, Cheonan, Korea in 2011. He is a Ph.D candidate of Computer Science Engineering at Korea University of Technology and Education, Korea. His main research is Ubiquitous computing, Wireless Sensor Network, Ad-hoc network, International Mobile Telecommunications-Advanced, Wireless SoC.

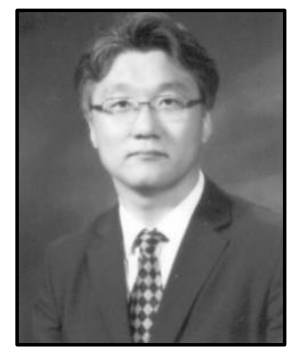

Kang-whan Lee, he received his B.S degree, electronics engineering from Hanyang University, Seoul, Korea, in 1983. The M.S degree in electronics engineering from Jungang University, Seoul, Korea in 1989. He worked Research Engineer in Electronics and Telecommunications Research Institute. He received a Ph.D candidate of electronics engineering from Jungang University, Seoul, Korea. $\mathrm{He}$ is an associate professor at Korea University of Technology and Education. His main research is Wireless Sensor Network, Ad-hoc network, International Mobile Telecommunications-Advanced, Wireless SoC. 